\title{
Correction to: Comparing the Preferences of Patients and the General Public for Treatment Outcomes in Type 2 Diabetes Mellitus
}

\author{
Published online: 4 November 2020 \\ C) Springer Nature Switzerland AG 2020

\section{Correction to: The Patient - Patient-Centered Outcomes Research https://doi.org/10.1007/s40271-020-00450-7}

Norah L. Crossnohere ${ }^{1,2} \oplus \cdot$ Sarah Janse ${ }^{1} \cdot$ Ellen Janssen $^{3} \cdot J^{\prime}$ John F. P. Bridges ${ }^{1,2}$

In the 2nd paragraph of the Introduction, the 2nd to last sentence previously read as

For instance, experience-based time trade-off and visual analog scale value sets for the EQ-5D-3L were recently published [16].

It should read as:

For instance, experience-based time trade-off and visual analog scale value sets for the EQ-5D-5L were recently published [16].

The original article can be found online at https://doi.org/10.1007/ s40271-020-00450-7.

Norah L. Crossnohere

norah.crossnohere@osumc.edu

1 Department of Biomedical Informatics, The Ohio State University College of Medicine, 1800 Cannon Drive, Columbus, OH 43210, USA

2 Department of Health, Behavior, and Society, Johns Hopkins Bloomberg School of Public Health, 624 N. Broadway, Baltimore, MD 21205, USA

3 Center for Medical Technology Policy, World Trade Center Baltimore, 401 East Pratt Street, Suite 631, Baltimore, MD 21202, USA 